brandenburg, stanley, and anatum, showed no reduction in viable count compared with MacConkey's medium. The growth of salmonellae was somewhat slower than on ordinary MacConkey agar but most produced colonies of $1 \mathrm{~mm}$ diameter at 22 hours and only five required incubation for 36 hours. Six of the 41 Salmonella species tested failed to grow (typhi, paratyphi $A$ and $C$, cholerasuis, gallinarum and pullorum) but all are rare causes of food poisoning in Britain. All the faecal flora was prevented from growing apart from occasional strains of lactose-fermenters and some paracolons. Inocula of one million organisms of Pseudomonas pyocyanea, E. coli, Proteus mirabilis, vulgaris and morgani, Klebsiella aerogenes, and Shigella sonnei were completely suppressed. The new medium was simple and reproducible from batch to batch.

An outbreak of $S$. enteritidis food poisoning amongst nursing staff allowed a comparison of the results from the new medium with those from deoxycholate-citrate agar (DCA). Each sample of faeces from 640 people was examined in two laboratories, one employing selenite $F$ and the new medium and another making orthodox use of first class DCA and selenite F. The total number of salmonellae isolated from the new medium was 36 compared with only 22 from DCA.

The new medium appeared to be a distinct advance on any other for the investigation of salmonella food poisoning.

\section{AN APPRECIATION OF THE BRUCELLIN SKIN TEST}

C.H.L. HOWELLS (Wolverhampton) The paper describes an epidemiological and serological survey of brucellin on volunteers. Each had venepuncture and received $0.1 \mathrm{ml}$ of brucellin (Olin, 1935) intradermally, using a disposable tuberculin syringe and needle. Details of age, occupation, consumption of unpasteurized milk, contact with cattle, and any history of brucellosis were noted. Participants suffering from brucellosis were excluded. Reactions were measured 48 hours later (positive tests $=10 \mathrm{~mm}$ erythema). Second venepuncture was done three weeks later to determine whether there had been any antibody stimulation. Agglutinations in phenol saline and mercaptoethanol, Coombs test, and complement-fixation tests were performed at Colindale, Truro, Northallerton, and Wolverhampton.

Proportion of positive reactions was greater in farming areas and increased with age. Consumption of unpasteurized milk and contact with cattle were associated factors. Four to seven per cent of healthy young adults might have been exposed to Brucella organism in the past, as positive skin reactions persisted after circulating antibody disappeared. Serological tests were more valuable than skin tests in assessing level of infection in community. In the survey, antibody was detected more easily with agglutination and Coombs tests than with mercaptoethanol and complement-fixation tests. This finding was expected in healthy volunteers since the latter tests indicate active infection and the former measure residual antibody.

It was concluded that the brucellin test is a poor indication of disease since many with positive tests did not possess antibody and some with negative tests did. Positive tests in farming areas were of little diagnostic $\underset{\overrightarrow{5}}{\stackrel{2}{*}}$

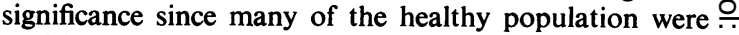
positive. Stimulation of antibody by brucellin was a further disadvantage. Caution therefore is needed in inter- $\stackrel{\oplus}{+}$ preting serological tests in anyone previously skin tested.

Brucellin appeared to be less useful than serological $\frac{\bar{\sigma}}{\bar{\Omega}}$ methods in epidemiology, while in diagnosis its use might $\frac{\bar{G}}{S}$ be entirely misleading.

AN ASSESSMENT OF SERUM ${ }^{57}$ CO CYANOCOBALAMIN AS AN INDEX OF VITAMIN B 12 ABSORPTION

D. DONALDSON AND P. T. LASCELLES (London) A technique $\overrightarrow{\vec{\omega}}$ has been developed for assaying accurately the low levels of ${ }^{57} \mathrm{Co}$ cyanocobalamin obtained in the serum during the $\frac{7}{0}$ conventional Schilling test. Emphasis is placed on the iv length of time (approximately two hours) required for obtaining reliable data in counts that are only slightly? above the background. The maximum radioactivity was found by serum tolerance tests to occur at eight hours. '่ Correlation between the eight-hour serum levels and 0 24-hr urinary excretion was studied in 96 patients and the data subsequently were subjected to mathematical analysis.

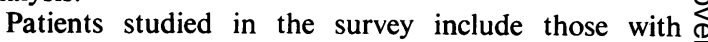
pernicious anaemia, with and without neurological $\frac{7}{3}$ involvement, malabsorption and postgastrectomy states, 믐 undiagnosed peripheral neuropathies, patients with hypopituitarism and myxoedema, and folate-deficient epilepto patients on treatment.

By serum counting it was possible clearly to demarcat; patients with pernicious anaemia from the norma controls, but pernicious anaemia (part two, with intrinsic factor), malabsorption syndrome and postgastrectomy patients, and folate-deficient epileptic patients on treatment fell into an intermediate range which could $\varrho$ not be differentiated from the normals.

A further group, namely, eight hypopituitary and myxoedematous patients on replacement therapy had significantly higher serum and urine levels than the normal controls. No obvious explanation was apparent.

The value of counting serum radioactivity in Schilling tests is shown to be of particular value where urine $\dot{0}$ collection is inaccurate, where there may be contamination of urine with extraneous radioactivity, and where there is the possibility that other isotopes have been given to the patient.

INVESTIGATION OF THE CELLULAR DEFECT IN VITAMIN $B_{12} \frac{7}{0}$ DEFICIENCY

D. G. CHALMERS (Cambridge) By flash labelling with tritiated thymidine freshly aspirated human bone marrow, $\mathrm{O}$ it is possible to determine, in individual cells, their $\mathrm{N}$ morphological category, their content of DNA, and $\sigma$ whether they were in DNA synthesis at the time of aspiration.

Previous investigations of pernicious anaemia have shown a failure of DNA synthesis in a proportion of cells? in this condition, and a pile up of cells in $\mathbf{G}_{\mathbf{2}}$.

In the knowledge that RNA synthesis, as measured by

. 